\title{
O GEOPROCESSAMENTO NA CONSTRUÇÃO DE CIDADES INTELIGENTES NO BRASIL
}

\author{
Vicente de Paula Sousa Júnior ${ }^{1}$ Universidade Federal do Piauí-UFPI
}

\section{RESUMO}

A busca pelo desenvolvimento sustentável passa pelo conceito de cidades inteligentes. $\mathrm{O}$ crescimento populacional urbano evidencia ainda mais a necessidade de que a gestão do município seja cada vez mais preocupada em solucionar problemas, além de prevê-los. O Geoprocessamento atua nessa área como suporte para criação da cidade digital, como também auxiliar no planejamento urbano e rural das cidades. Esse artigo tem como objetivo de elencar como o uso do Geoprocessamento pode auxiliar na construção das Cidades Inteligentes no Brasil, de acordo com a legislação vigente e suas particularidades. Consta-se que é importante que o Plano Diretor dos municípios esteja alinhado com os objetivos da sustentabilidade e desenvolvimento de uma gestão mais inteligente, contemplando as políticas de Georreferenciamento de Imóveis Rurais, Cadastro Territorial Multifinalitário - CTM e integrando dados de maneira que o acesso seja fácil, como também evitando a duplicidade. Ao final, a Cidade ganha tecnologia para o planejamento de seu desenvolvimento, arrecadação de impostos, prevenção de desastres, integração de dados de várias áreas que contribuem não só para a construção da Cidade Inteligente, mas com o desenvolvimento sustentável.

Palavras-Chave: GIS. Smart Cities. Estatuto das Cidades. SINTER. INDE.

\section{INTRODUÇÃO}

A humanidade passa por dois grandes fenômenos que são a revolução digital com o avanço tecnológico e a expansão global urbana (BOUSKELA et al., 2016). Segundo Queiroz (2018), o crescimento da população urbana aliada à busca em construir um desenvolvimento sustentável para cidade, utilizando-se da tecnologia de informação para detectar e resolver problemas urbanos sugere a concepção de um novo conceito, do inglês Smart City, que significa Cidade Inteligente. "Por um viés mais dirigido, pode-se conceituar como "cidade inteligente", o município cujo Governo Local se apropria das geotecnologias da informação, instrumentalizando sua gestão e planejamento, tornando a governança mais colaborativa e inclusiva." (QUEIROZ, 2018, p. 27).

O planejamento, gerenciamento e governança, ou seja, gestão das cidades de forma sustentável tem por princípio maximizar as oportunidades econômicas e minimizar os danos ambientais, sendo esses os desafios que o mundo enfrentará nesse século (BOUSKELA et al.,

\footnotetext{
${ }^{1}$ Mestrando, Desenvolvimento e Meio Ambiente, Universidade Federal do Piauí, vicentepsj@outloqkBẈ978-65-80968-11-4
} DOI: $10.17648 /$ cbcs-2019-110661 
2016). Para isso pode-se utilizar o Geoprocessamento como alicerce para atingir o desenvolvimento da Cidade Inteligente, e consequentemente, cidade sustentável.

Câmara e Davis (2004) abordam que o termo refere-se à área de conhecimento que se utiliza de técnicas computacionais e matemáticas que estão voltadas ao tratamento de informações geográficas de diversas áreas como Cartografia, Análise de Recursos Naturais, Transportes, Comunicações, Energia e Planejamento Urbano e Regional. Para que a cidade alcance o patamar de Cidade Digital, que corresponde à utilização de tecnologias, como o SIG para solucionar problemas urbanos e rurais e criar infraestruturas sociotécnicas (REZENDE et al., 2014).

O presente artigo objetiva elencar dentro das normas, legislação, políticas públicas e ferramentas brasileiras que promovam o desenvolvimento de Cidades Inteligentes os tópicos em que o Geoprocessamento pode atuar para construção das mesmas. Dentre esses aspectos pode-se destacar o Estatuto das Cidades, Georreferenciamento de Imóveis Rurais (Cidades Inteligentes não são estruturadas apenas na área urbana das cidades), Infraestrutura Nacional de Dados EspaciaisINDE, Cadastro Territorial Multifinalitário-CTM, Sistema Nacional de Gestão de Informações Territoriais - SINTER, NBR 13.133 e NBR 14.166.

\section{METODOLOGIA}

Para que o objetivo do artigo fosse atingido foi utilizada para o método que consiste na revisão do referencial teórico em bases de periódicos indexados, utilizando-se ainda de uma pesquisa que demonstre as leis, estatutos e políticas públicas que estão diretamente ligadas ao assunto que envolve as cidades inteligentes no Brasil e a atuação do Geoprocessamento.

\section{O ESTATUTO DAS CIDADES}

Este é uma das principais meio para que o controle e regras sobre as cidades sejam realizados de maneira correta. O Estatuto das cidades foi instituído por meio da Lei no 10.257/01, estabeleceu as diretrizes gerais da política urbana e deu outras providencias. Além disso, regulamenta os artigos 182 e 183 da Constituição Federal (BRASIL, 2001).

Segundo Mukai (2001), o Estatuto é constituído de cinco capítulos, mas o tratamento sobre o Plano Diretor dos municípios e sua obrigatoriedade é a parte mais relevante do mesmo. $O$ autor segue comentando sobre as diretrizes gerais e aponta que o Estatuto das Cidades é uma garantia a cidades sustentáveis, direito a terra urbana, infraestrutura urbana, saneamento, dentre outras pautas. 


\section{CONGEESSOC CIENCIAESOCIEDADE

Segundo Carvalho (2019), torna-se necessário que o município defina um objetivo, nesse caso, torna-se uma cidade inteligente, logo adiante é necessário que o Plano Diretor do município contemple os passos para que isso seja concretizado. Além disso, a busca por parcerias que ajudem a alcançar esse objetivo. A autora explica isso baseada na obra de Napoleão Hill "Lei do Triunfo".

O Geoprocessamento utiliza-se do estatuto para garantir a aplicação do planejamento do crescimento urbano, auxiliando no cálculo do IPTU, que geram arrecadação para o município poder investir em diversas áreas, e consequentemente, melhorar a qualidade de vida da população. Afinal, a arrecadação pelos municípios de forma individual demonstra uma independência em relação aos recursos repassados pelos governos estadual e federal.

\section{GEORREFERENCIAMENTO DE IMÓVEIS RURAIS}

Relacionado com vários outros marcos legais, como o Estatuto da Terra (Lei no 4.504/1964), Código Florestal Brasileiro criado em 1965, mas que foi alterado e atualizado pela Lei no 12.727/2012, dentre outras leis, decretos, resoluções e normas técnicas. O Georreferenciamento de Imóveis está diretamente relacionado aos parágrafos 3 e e 4으, do artigo 176, e o parágrafo 3ำ do artigo 225 da Constituição Federal, da Lei no 6.015/1973, incluídos pela Lei $n$ ㅇ $10.267 / 2001$.

O Instituto Nacional de Colonização e Reforma Agrária-INCRA, é responsável por analisar os levantamentos realizados para fins do Georreferenciamento de imóveis rurais. Por isso, lançou uma Norma Técnica que já está na 3o edição para orientar esses levantamentos. O profissional habilitado deve realizar a coleta de dados seguindo o padrão estabelecido para que os órgãos tenham o fiel conhecimento da malha territorial do país (INCRA, 2013).

\section{INFRAESTRUTURA NACIONAL DE DADOS ESPACIAIS - INDE}

O governo brasileiro designou para a Comissão Nacional de Cartografia - CONCAR, que fosse elaborado com um prazo de 180 dias, o Plano de Ação e implementação da INDE. A INDE foi instituída pelo Decreto $\mathrm{n}$ - 6.666/2008 objetivando promover o ordenamento adequado na geração, armazenamento, disseminação e no uso dos dados geoespaciais em todos os níveis de governo, promovendo a utilização na produção dos dados nas instituições públicas, seguindo as 
normas e padrões estabelecidos pela CONCAR (BRASIL, 2008). A Infraestrutura de Dados Espaciais é definida por Maguirre e Longley (2005):

Tecnologias, políticas, normas, recursos humanos e atividades relacionadas, necessárias para aquisição, processamento, distribuição, utilização, manutenção e preservação de dados espaciais em todos os níveis de governo, os setores privados e sem fins lucrativos e universidades (MAGUIRRE E LONGLEY, 2005, p. 5).

A Infraestrutura de Dados Espaciais objetiva que a coleta de dados redundantes seja suprimida, uma vez que os dados são compartilhados por meio de padrões abertos a diversas instituições. Uma vantagem da Infraestrutura de Dados Espaciais é a facilidade de manuseio dos dados em diversas escalas (global, regional, nacional, estadual, local e organizacional), ou seja, o compartilhamento de informações e dados pode ser feito entre países, nacional, que é onde encontramos a INDE. Além disso, os dados ainda podem ser dispostos de maneira estadual, municipal e entre organizações públicas e privadas (INDE, 2019).

\section{CADASTRO TERRITORIAL MULTIFINALITÁRIO - CTM}

A implantação do CTM é ponto forte para uma boa gestão urbana. Segundo Erba e Piumentto (2013), é o instrumento fundamental para definir o planejamento de políticas que se referem ao solo urbano. Queiroz (2018) complementa que os dados de diversas áreas contribuem para o monitoramento e gerenciamento das cidades, auxiliando na definição do plano de estratégias para o financiamento urbano, que consegue prever impactos das intervenções antes e depois de serem realizadas.

É importante entender o que faz o cadastro territorial ser multifinalitário, Araújo e Silva (2014) destacam que o cadastro só será reconhecido como multifinalitário quando sua finalidade não for apenas para fins tributários, como IPTU, ainda para fins fiscais e jurídicos. Já que o cadastro que contempla apenas esses aspectos é caracterizado como tradicional.

Por meio da multifinalidade, busca-se atender às necessidades da Administração Pública e melhorar a gestão municipal, não só sob os aspectos financeiros e de arrecadação, mas contemplando também as dimensões social, ambiental, física e jurídica que o instrumento pode potencializar, colaborando para a efetivação de sua autonomia e para a ampliação da transparência nas ações do poder público, fundamentos essenciais para a construção de uma cidade democrática e com justiça social. (CUNHA; ERBA, 2010, p.11). 
Os resultados obtidos por análises dos dados coletados pelo CTM são diversos, como uso e ocupação do solo, mapas com zoneamento urbano, mapas que seguem o padrão do Plano Diretor, planos de mobilidade urbana, saneamento, transporte público, resíduos sólidos, habitação entre outros. Já no aspecto financeiro, "possibilita a modernização da gestão dados dos cadastros imobiliário, mobiliário, de logradouros; geração e atualização da Planta Genérica de Valores - PGV; além da possibilidade de monitoramento e espacialização da dívida ativa." (Queiroz, 2018, p.33).

\section{SISTEMA NACIONAL DE GESTÃO DE INFORMAÇÕES TERRITORIAIS - SINTER}

O SINTER foi instituído pelo Decreto no 8.764/2016, que é ferramenta de gestão pública que objetiva realizar a integração de um banco de dados espaciais, o fluxo dinâmico de dados jurídicos produzidos pelos serviços de registros públicos ao fluxo de dados fiscais, cadastrais e geoespaciais de imóveis urbanos e rurais. Tabak e Dayrell (2017) dizem que o sistema foi criado para resolução do problema de integração dos dados relacionados ao vários cadastros e registros territoriais do Brasil. Ainda segundo os autores, a ideia de um sistema único para armazenamento desses dados diminui os custos em buscar informações, além de aumentar a segurança jurídica.

O sistema possibilita uma interoperabilidade, que é uma característica importante para gestão e aplicação de políticas públicas. Ao final, informações territoriais e do CTM unidas em um ambiente SIG podem gerar mapas com análises que abrangem diversos campos como o ambiental, social, econômico entre outros. Dessa forma, tem-se uma visão macro que possibilita o entendimento local dos municípios na área urbana e rural (TABAK e DAYRELL, 2017).

\section{UTILIZAÇÃO DA NBR 13.133 E NBR 14.166}

A Associação Brasileira de Normas Técnicas - ABNT estabelece duas normas importantes para o desenvolvimento das cidades inteligentes, que é a NBR 13.133, que estabelece as condições exigíveis para execução de levantamentos topográficos que são destinados a obtenção de conhecimento geral do terreno, informações sobre o terreno destinado a desenvolvimento de estudos preliminares de projetos, assim como informações destinadas a projetos executivos (ABNT, 1996). 
A NBR 14.166 é destinada a normatizar as condições exigíveis para a implantação de uma Rede de Referência Cadastral, que é necessária para estabelecer a infraestrutura adequada para dar apoio geodésico e topográfico que proporcione a padronização de levantamentos topográficos (ABNT, 1998). Ainda tem-se que:

Esta rede, portanto, deve apoiar tanto as atividades cadastrais a serem representadas no Plano Topográfico Local, em escala 1:1 000 até 1:1, como os levantamentos destinados à cartografia, inclusive à sistemática, representados em projeção UTM usualmente em escala 1:2 000 ou menores. (ABNT, 1998, p.1)

Pode-se dizer que as normas se complementam e são ferramentas de captação de dados que devem ser integrados tanto ao CTM como ao SINTER. Um sistema de leis e estatutos é fundamental para que o desenvolvimento de setores da sociedade seja possível, por exemplo, setor energético necessita de estabilidade regulatória para seu crescimento e desenvolvimento, assim como o setor de transporte público. Isso ocorre pelo fato dos setores necessitarem não apenas do esforço do sistema público, mas de investimentos privados. Ainda, é de conhecimento o desenvolvimento de uma NBR voltada para o Cadastro Urbano que se assemelha com o Georreferenciamento de Imóveis Rurais.

\section{CONSIDERAÇÕES FINAIS}

Constata-se que no campo regulatório a atuação do Geoprocessamento está bem definida em como ajudar a transformação da conjuntura atual das cidades brasileiras para alcançar o patamar de cidades inteligentes. Destacando o Plano Diretor de Ordenamento do Território PDOT como peça fundamental para os municípios, por direcionar e planejar o espaço dos municípios no âmbito urbano e rural. É essencial que o PDOT seja atualizado e tenha os princípios da Cidade Digital integralizados.

Uma cidade que aplica e utiliza o Geoprocessamento como meio e apoio ao planejamento e ordenamento territorial evita gasto com duplicidade de dados, obtenção de dados primários do seu território como curvas de nível, classe do solo entre outros. Obtendo essas informações em formato digital que possibilitam a análise e modelagem de cenários para o município. Essas informações que antes poderiam ter alto custo pelo fato da Aerofotogrametria necessitar de aviões equipados por câmeras, atualmente é substituído por levantamentos realizados por drones. 
É necessário ainda que os municípios se organizem de maneira a criar sua própria infraestrutura de dados e um órgão que responda diretamente a realizar a coleta, armazenamento, análise e modelagem dos dados. Esse órgão ficaria responsável por gerenciar um banco de dados geográficos de várias áreas de atuação municipal, ou seja, desde a saúde até as obras de infraestrutura, pois a integralização desses dados possibilitam análises espaciais que modelam cenários em que a prevenção de problemas e planejamento de ações para melhoria à população. Essa capacidade de integração de dados também necessita que a equipe do órgão seja multidisciplinar, pois o Geoprocessamento possui vários campos de atuação e uma equipe que tenha a capacidade de analisar cenários de várias perspectivas torna-se um diferencial para implantação e construção de uma cidade inteligente que almeja atingir as metas e objetivos do desenvolvimento sustentável.

\section{REFERÊNCIAS}

ABNT. NBR 13.133. 1996. Disponível em: <http://www.carto.eng.uerj.br/cdecart/download/NBR13133.pdf>. Acesso 02 de set. 2019.

ABNT. NBR 14.166. 1998. Disponível em: <http://www.carto.eng.uerj.br/cdecart/download/NBR14166.pdf>. Acesso 02 de set. 2019.

ARAÚJO, F. A.; SILVA, C. N. O cadastro territorial multifinalitário (CTM): (multi) finalidades e perspectivas para o ordenamento territorial urbano. Revista Formação, n.21, volume 2, 2014, p. 23-48. Disponível em: http://revista.fct.unesp.br/index.php/formacao/article/viewArticle/2830 Acesso 02 set. 2019.

BOUSKELA, M.; CASSEB, M.; BASSI, S.; DE LUCA, C.; FACCHINA, M. Caminho para as smart Cities: da gestão tradicional para a cidade inteligente. BID, 2016. Disponível em: $<$ https://publications.iadb.org/publications/portuguese/document/Caminho-para-as-smart-citiesDa-gest\%C3\%A3o-tradicional-para-a-cidade-inteligente.pdf>. Acesso 02 de set. 2019.

BRASIL. Decreto n. 6.666 de 27 de Novembro de 2008. Disponível em: <http://www.planalto.gov.br/ccivil_03/_Ato2007-2010/2008/Decreto/D6666.htm>. Acesso 02 de set. 2019.

BRASIL. Lei $\mathbf{n} . \mathbf{1 0 . 2 5 7}$ de $\mathbf{1 0}$ de Julho de 2001. Disponível em: <http://www.planalto.gov.br/ccivil_03/leis/leis_2001/l10257.htm>. Acesso 02 de set. 2019.

CÂMARA, G.; DAVIS, C. Introdução à Ciência da Geoinformação: Capitulo 1 - Apresentação. INPE, 2004. Disponível em: <http://www.dpi.inpe.br/gilberto/livro/introd/index.html>. Acesso 02 de set. 2019. 
CARVALHO, G. A. 10 Projetos GIS para Cidades Inteligentes. Webinar: 25 de Julho de 2019. Disponível em:

<http://events.genndi.com/r/3/0/replay/169105139238448981/2c12056719/0/134016518>.

CUNHA, E. M. P.; ERBA, D. A. Manual de Apoio - CTM: Diretrizes para criação, instituição e atualização do cadastro territorial multifinalitário nos municípios brasileiros. Brasília: Ministério das Cidades, 2010. Disponível em:

<http://www.capacidades.gov.br/media/doc/acervo/c4924c559c0b1b95a8ad38c47fda4799.pdf>. Acesso 03 de set. 2019.

ERBA, D. A.; PIUMETTO, M. Catastro Territorial Multifinalitario. Lincoln Institute of Land Policy, 2013. Disponível em: <https://www.lincolninst.edu/sites/default/files/pubfiles/erba-wp14de1spfull_0.pdf>. Acesso 02 de set. 2019.

INCRA. Norma Técnica para Georreferenciamento de Imóveis Rurais. 3 ed. 2013. Disponível em: $<$ http://www.incra.gov.br/estrutura-fundiaria/regularizacao-fundiaria/certificacao-de-imoveisrurais/file/1575-norma-tecnica-para-georreferenciamento-de-imoveis-rurais-3-edicao>. Acesso 02 de set. 2019.

INDE. Apresentação. Copyright 2019. Disponível em: <https://inde.gov.br/Inde/Apresentacao>. Acesso 03 de set. 2019.

MAGUIRE, D. J.; LONGLEY, P. A. The emergence of geoportals and their role in spatial data infrastructures. Computers, Environment and Urban Systems. v. 29, p. 3-14, 2005. Disponível em: <http://citeseerx.ist.psu.edu/viewdoc/download?doi=10.1.1.91.742\&rep=rep1\&type=pdf $>$. Acesso 03 de set. 2019

MUKAI, T. O Estatuto da Cidade. Revista de Direito Administrativo, Rio de Janeiro, v. 225, p. 343348, jul. 2001. ISSN 2238-5177. Disponível em: <http://bibliotecadigital.fgv.br/ojs/index.php/rda/article/view/47581>. Acesso 05 de set. 2019.

QUEIROZ, A. O. Cidades inteligentes: o papel do cadastro territorial Multifinalitário como ferramenta de gestão e planejamento. Rev. Nacional de Gerenciamento de Cidades. v.06, n.37, 2018. Disponível em:

<http://www.amigosdanatureza.org.br/publicacoes/index.php/gerenciamento_de_cidades/article /view/1767>. Acesso 02 de set. 2019.

REZENDE, D.A.; MADEIRA, G. S.; MENDES, L. S.; BREDA, G. D.; ZARPELÃO, B. B.; FIGUEIREDO, F. C. Information and Telecommunications Project for a Digital City: A Brazilian case study. Telematics and Informatics, v.31, n.1. p.98-114. 2014.

TABAK, B. M.; DAYRELL, C. C. UMA ANÁLISE ECONÔMICA DO SISTEMA NACIONAL DE INFORMAÇÕES TERRITORIAIS (SINTER): UM PROBLEMA DE DIREITO DE PROPRIEDADE. Rev. de Direito da Cidade. v. 09, n. 4. 2017. Disponível em: <https://www.epublicacoes.uerj.br/index.php/rdc/article/view/29199/21996>. Acesso 03 set. 2019. 